\title{
Correlation between the Efficacy of Lamotrigine and the Serum Lamotrigine Level during the Remission Phase of Acute Bipolar II Depression: A Naturalistic and Unblinded Prospective Pilot Study
}

\author{
Akiyoshi Kikkawa, ${ }^{a, b}$ Yoshihisa Kitamura, ${ }^{*, a}$ Tetsuya Aiba, ${ }^{c}$ Koichi Hiraki, ${ }^{b}$ and Toshiaki Sendo ${ }^{a}$ \\ ${ }^{a}$ Department of Clinical Pharmacy, Graduate School of Medicine, Dentistry, and Pharmaceutical Sciences, \\ Okayama University; 2-5-1 Shikata-cho, Kita-ku, Okayama 700-8558, Japan: ${ }^{b}$ Department of Pharmacy, \\ Hiroshima City Hospital; 7-33 Moto-machi, Naka-ku, Hiroshima 730-8518, Japan: and ${ }^{c}$ Department of Clinical \\ Pharmacokinetics and Therapeutics, Graduate School of Medicine, Dentistry, and Pharmaceutical Sciences, \\ Okayama University; 2-5-1 Shikata-cho, Kita-ku, Okayama 700-8558, Japan. \\ Received September 12, 2016; accepted December 27, 2016
}

Lamotrigine has acute antidepressant effects in patients with bipolar disorder. However, there is little information regarding appropriate serum levels of lamotrigine and the time until remission after the start of lamotrigine therapy in patients with bipolar II depression. This was a naturalistic and unblinded prospective pilot study. Twelve patients' depressive symptoms were evaluated using the Montgomery-Åsberg Depression Rating Scale (MADRS) at the start of treatment and at the time of remission, and blood samples were obtained at the time of remission. Mahalanobis distance was used to analyze the relationship between the MADRS improvement rate and the serum lamotrigine level. Furthermore, we calculated the Spearman's rank correlation coefficient for the relationship between the MADRS improvement rate and the serum lamotrigine level, and produced box plots of the serum lamotrigine level at remission and the time until remission. The Mahalanobis distance for the patient that was co-administered lamotrigine and valproic acid differed significantly from those of the other patients $(p<0.001)$. There was no linear relationship between the serum lamotrigine level and the MADRS improvement rate among the patients that did not receive valproic acid. The median time from the start of lamotrigine therapy until remission was 6 weeks. The serum lamotrigine level does not have an important impact on the acute therapeutic effects of lamotrigine on bipolar II depression. In addition, we consider that different treatment options should be considered for non-responders who do not exhibit any improvement after the administration of lamotrigine for approximately 6 weeks.

Key words lamotrigine; serum level; bipolar II depression; Montgomery-Åsberg Depression Rating Scale; Mahalanobis distance

Bipolar disorder is a recurrent psychiatric disorder that is characterized by a diverse range of symptoms, including manic, depressive, and mixed symptoms. According to the Diagnostic and Statistical Manual of Mental Disorders, 4th Edition, Text Revision (DSM-IV-TR), the lifetime community prevalence of bipolar II disorder is $0.5 \%$, but recent studies have reported lifetime prevalence rates of around $1.5-2 \% .^{1-4)}$ The course of the illness is characterized by recurrent episodes of hypomania and depression interspersed with periods of euthymia. Patients are symptomatic for $>50 \%$ of the time, with depression of varying severity being the most common symptom. $^{2)}$

Lamotrigine has demonstrated acute antidepressant effects in patients with bipolar disorder, ${ }^{5)}$ and the use of lamotrigine alone or in combination with other psychotropic drugs was found to be well tolerated and effective during the management of treatment-resistant bipolar II depression. ${ }^{6)}$ In addition, the augmentation of antidepressant therapy with lamotrigine was shown to be effective against treatment-resistant unipolar depression. ${ }^{7)}$ Furthermore, van der Loos et al. reported that lamotrigine was effective and safe when it was used as an add-on treatment to lithium during the acute treatment of bipolar depression. ${ }^{8)}$ In 2011, lamotrigine was approved for use as a prophylactic treatment in Japan, particularly for relapsed bipolar disorder.

The side effects of lamotrigine are reported to include drug- induced skin eruptions, balance problems, drowsiness, and dizziness. ${ }^{9)}$ Drug-induced skin eruptions can lead to serious eruptions, such as Steven-Johnson syndrome or toxic epidermal necrolysis. ${ }^{10,11)}$

There is disagreement in the literature regarding the utility of therapeutic drug monitoring during lamotrigine treatment. It was reported that no clear-cut relationships exist between the serum lamotrigine concentration and the clinical response or toxicities in epilepsy patients. ${ }^{12-14)}$ In addition, only a few attempts have been made to determine the optimal effective serum lamotrigine level for treating depressive symptoms in patients with bipolar II disorder. To start to address this issue, we have conducted a naturalistic and unblended prospective pilot study.

It has been suggested that lamotrigine metabolism might be markedly affected by the concomitant administration of valproic acid and/or carbamazepine. ${ }^{15)}$ Therapeutic drug monitoring is usually required to optimize psychotropic therapy, such as that involving valproic acid, carbamazepine, or lithium. Valproic acid is known to be a potent inhibitor of lamotrigine metabolism; i.e., it causes a three-fold increase in the elimination half-life of lamotrigine. ${ }^{16,17)}$ The combined use of lamotrigine and valproic acid therefore results in significantly higher systemic lamotrigine levels and an increased risk of severe side effects. ${ }^{18)}$ In addition, it is known that during combination therapy lamotrigine levels vary greatly according to the 
concomitant drug administered. However, the optimal serum levels of lamotrigine and the time required to achieve remission during lamotrigine therapy are unclear in patients with bipolar disorder, especially those with bipolar II depression. Therefore, the purpose of this study was to investigate the relationship between the serum lamotrigine level and the time required to achieve depression remission during lamotrigine treatment in patients with bipolar II disorder.

\section{MATERIALS AND METHODS}

Sample Collection This naturalistic and unblinded prospective pilot study was performed at the Department of Psychiatry of Hiroshima City Hospital, Japan, between April 1, 2013, and August 31, 2014 (the patients' demographic and clinical data are summarized in Table 1). The inclusion criteria were being an inpatient that had been diagnosed with bipolar II disorder (DSM-IV-TR) by an experienced psychiatrist, but had not been prescribed lamotrigine.

Study Design and Measures The patients' depressive symptoms were evaluated using the Montgomery-Åsberg Depression Rating Scale (MADRS). The time of remission was defined as the point at which the patient achieved a MADRS score of 12 points and the treating psychiatrist had stopped increasing the lamotrigine dose. Blood samples were collected at the time of remission, and all samples were obtained at $12 \mathrm{~h}$ after the administration of lamotrigine. The patients' serum lamotrigine levels were measured using a HPLC system by SRL Co. (Tokyo, Japan). Lamotrigine therapy was started at a dose of $25 \mathrm{mg}$ a day, and the dose was gradually increased every two weeks. The co-administration of valproic acid was started at a dose of $25 \mathrm{mg}$ every two days, and the dose was gradually increased every two weeks. The co-administration of carbamazepine was started at a dose of $50 \mathrm{mg}$ a day, and

Table 1. Demographic and Clinical Characteristics

\begin{tabular}{ll}
\hline \hline Characteristics & \\
\hline Gender, $N(\%)$ & $8(67)$ \\
Female & $4(33)$ \\
Male & $55.0 \pm 15.3$ \\
Age, mean & $30.2 \pm 9.5$ \\
Baseline MADRS score & $4.7 \pm 4.2$ \\
Final MADRS score & $0.6 \pm 0.2$ \\
MADRS improvement rate & \\
Combined use of a mood stabilizer, $N(\%)$ & $1(8)$ \\
Valproic acid & $2(17)$ \\
Carbamazepine & $9(75)$ \\
Other & \\
Concentration dose ratio $(\mu \mathrm{g} / \mathrm{mL} / \mathrm{mg})$ & 0.072 \\
$\quad$ Combined use of valproic acid & 0.016 \\
Combined use of carbamazepine & 0.030 \\
Other & $12.3 \pm 9.8$ \\
Duration of illness (years) & $1(8.3)$ \\
Antidepressants being taken, $N(\%)$ & $2(16.7)$ \\
SSRI & $1(8.3)$ \\
Mirtazapine & \\
Tetracyclics & $8(66.7)$ \\
Atypical psychotics being taken, $N(\%)$ & \\
Quetiapine & \\
\hline
\end{tabular}

\footnotetext{
Abbreviation: SSRI=Selective serotonin reuptake inhibitors.
}

the dose was gradually increased every two weeks. These dosage methods are recommended by GlaxoSmithKline. Side effects were appropriately confirmed by psychiatrists and pharmacists.

Statistical Analysis The relationship between the patients' MADRS improvement rates and their serum lamotrigine levels on the day of the induction of remission was elucidated by calculating the Mahalanobis distance. The MADRS improvement rate was calculated by dividing the difference between the MADRS score on the day on which lamotrigine therapy was started and the MADRS score on the day of remission by the number of days required to achieve remission. Thus, the MADRS improvement rate of a patient whose symptoms improved quickly would be higher than that of a patient who recovered slowly. As the Mahalanobis distance can be utilized to determine a suitable boundary for identifying individuals with different characteristics to the other subjects, each of the 12 patients was subjected to examinations designed to determine whether they differed from the others based on the Mahalanobis distance from the center of the data distribution. Thus, after excluding the data for a given patient, the Mahalanobis distance for the relationship between the MADRS improvement rate and the serum lamotrigine level was calculated using the data for the other 11 patients, and $p$-values of $<0.001$ were considered to be statistically significant. In cases in which the patients' data fell outside of the boundary, the patient was considered to be different from the others. After calculating the Mahalanobis distance and excluding heterogeneous subjects, the correlation between the MADRS improvement rate and the serum lamotrigine level was assessed using Spearman's correlation coefficient analysis. The data relating to the patients' serum lamotrigine levels and the time required to achieve remission are expressed using box and whisker plots. Data are expressed as mean \pm standard deviation (S.D.) values.

Ethics All of the subjects gave their informed consent, and the study protocol was approved by the ethics committee of Hiroshima City Hospital (No. 24-94). After informed consent had been obtained, the patients had their MADRS scores evaluated and underwent pharmacokinetic analysis.

\section{RESULTS}

Subjects The patients' characteristics are shown in Table 1. The study comprised 12 bipolar II depression patients who had their MADRS scores evaluated and underwent pharmacokinetic analysis (Tables 1, 2). All of the patients had been diagnosed with bipolar II disorder (DSM-IV). The patients included 4 males and 8 females, and their mean age was 55.0 \pm 15.3 . The patients' mean baseline MADRS score was $30.2 \pm 9.5$, and their mean final MADRS score was 4.7 \pm 4.2 . The mean lamotrigine dose $(\mathrm{mg})$ at the time of remission was $91.7 \pm 28.9$, the mean time until remission was $6.0 \pm 1.7$ weeks, and the mean serum lamotrigine level at the time of remission was $2.8 \pm 1.7 \mu \mathrm{g} / \mathrm{mL}$. As for the combination of lamotrigine with other mood stabilizers, valproic acid was used in one patient, carbamazepine was administered to 2 patients, and lithium was given to 3 patients. Concentration dose ratios (C/D ratios) $(\mu \mathrm{g} / \mathrm{mL} / \mathrm{mg})$ of 0.072 (combined use of valproic acid, $n=1$ ), 0.030 (combined use of other drugs, $n=9$ ), and 0.016 (combined use of carbamazepine, $n=2$ ) were 
seen. The mean duration of the patients' illness was $12.3 \pm 9.8$ years. Most patients were taking atypical psychotic drugs. One patient each was taking serotonin reuptake inhibitors and a tetracyclic antidepressant, and two patients were taking mirtazapine. During the study period, none of the patients had recently started using antidepressants, and none of the patients had recently started using psychotropic drugs, except hypnotics. Furthermore, valproic acid and carbamazepine can affect the metabolism of lamotrigine, but the doses of valproic acid and carbamazepine were not changed during the study period in any of the patients that were prescribed these drugs. None of the patients developed marked side effects during the study.

Correlation between the Patients' Serum Lamotrigine Levels and the MADRS Improvement Rate The correlation between the patients' serum lamotrigine levels at the time of remission and the overall MADRS improvement rate for all

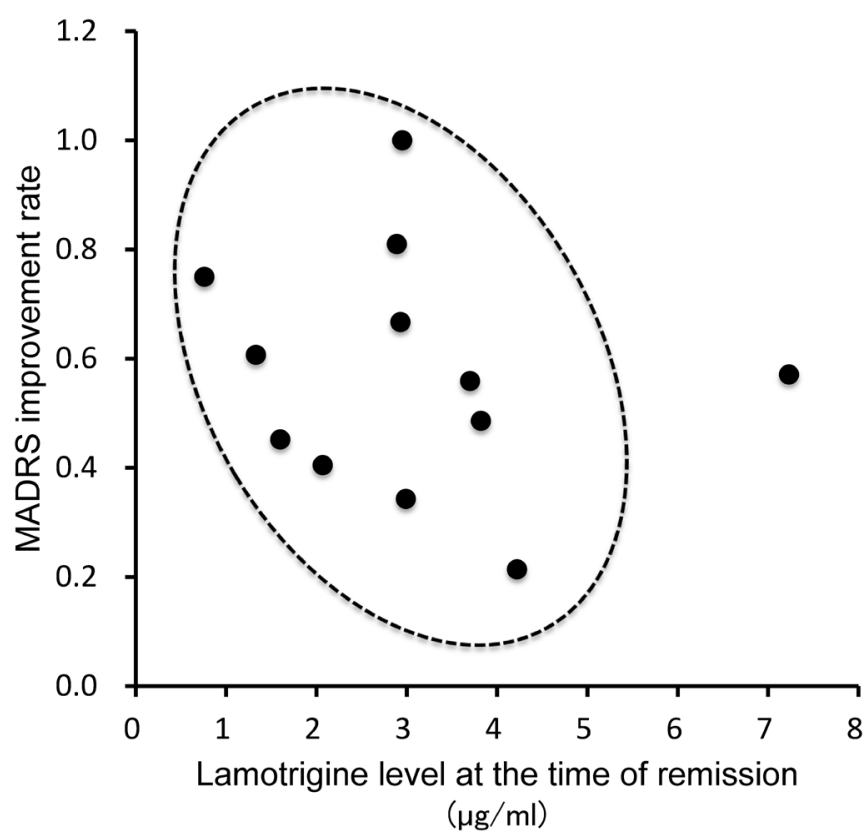

Fig. 1. Relationship between the Patients' Serum Lamotrigine Levels at the Time of Remission and the MADRS Improvement Rate According to the Mahalanobis Distance

The dotted line represents the $95 \%$ confidence interval. patients was determined (Fig. 1, Table 2). The Mahalanobis distance for the patient that was co-administered lamotrigine and valproic acid differed significantly from those of the other patients $(p<0.001)$. Thus, the data for the patient that received combination treatment involving lamotrigine and valproic acid was excluded from the analysis. After excluding this patient, no correlation was detected between the patients' serum lamotrigine levels at the time of remission and the MADRS improvement rate $(r=-0.2528, y=-0.0557 x+0.7674$, $R^{2}=0.06392$ ). Furthermore, the Spearman's rank correlation coefficient for this relationship was not significant among the remaining patients. The patients' maximum, minimum, and median serum lamotrigine levels at the time of remission were $4.22,0.76$, and $2.35 \mu \mathrm{g} / \mathrm{mL}$, respectively, and the associated third quartile and first quartile values were 2.94 and $1.61 \mu \mathrm{g} / \mathrm{mL}$, respectively (Fig. 2A). The maximum, minimum, and median times until remission were 7.5 , 4, and 6 weeks, respectively. The third and first quartile values of the time until remission were 6 weeks and 5 weeks, respectively (Fig. 2B). There was one case in which the time until remission was 10 weeks, but it was considered to be an outlier.

\section{DISCUSSION}

The present study suggests that there is no correlation between the MADRS improvement rate and the serum lamotrigine level in bipolar II disorder patients with depressive symptoms. Furthermore, we found that remission was achieved after 6 weeks of lamotrigine treatment and that the maximum serum lamotrigine level at the time of remission was $4.2 \mu \mathrm{g} / \mathrm{mL}$.

Lamotrigine appears to be particularly useful as a maintenance treatment; i.e., at decreasing depressive bipolar symptoms and preventing relapse. The efficacy of lamotrigine as a maintenance therapy was established in long-term studies involving subjects with bipolar I disorder who had been manic or depressed, as well as in subjects with rapid-cycling bipolar II disorder. ${ }^{19-21)}$ Furthermore, a meta-analysis did not detect any difference in the response to lamotrigine between the bipolar I and II disorder subgroups. ${ }^{22)}$ On the other hand, the therapeutic window of lamotrigine treatment for mood disorders (including bipolar I and II and major depressive dis-

Table 2. Clinical Details of Acute Bipolar II Depression Patients That Were Treated with Lamotrigine

\begin{tabular}{|c|c|c|c|c|c|c|c|c|c|c|}
\hline Patient & Gender & Age & $\begin{array}{l}\text { Lamotrigine } \\
\text { level }(\mu \mathrm{g} / \mathrm{mL}) \\
\text { at the time of } \\
\text { remission }\end{array}$ & $\begin{array}{l}\text { Lamotrigine } \\
\text { dose }(\mathrm{mg}) \text { at } \\
\text { the time of } \\
\text { remission }\end{array}$ & $\begin{array}{l}\text { Time until } \\
\text { remission } \\
\text { (weeks) }\end{array}$ & $\begin{array}{l}\text { Baseline } \\
\text { MADRS }\end{array}$ & $\begin{array}{c}\text { Final } \\
\text { MADRS }\end{array}$ & $\begin{array}{c}\text { MADRS } \\
\text { improvement } \\
\text { rate }\end{array}$ & $\begin{array}{c}\text { Mahalanobis } \\
\text { distance }\end{array}$ & $\begin{array}{c}\text { Concomitant mood } \\
\text { stabilizers }\end{array}$ \\
\hline 1 & $\mathrm{~m}$ & 75 & 1.60 & 100 & 6 & 22 & 3 & 0.452 & 1.091 & Carbamazepine \\
\hline 2 & $\mathrm{f}$ & 32 & 3.82 & 150 & 10 & 45 & 11 & 0.486 & 1.121 & None \\
\hline 3 & $\mathrm{~m}$ & 42 & 1.33 & 50 & 4 & 18 & 1 & 0.607 & 0.970 & Lithium \\
\hline 4 & $\mathrm{~m}$ & 37 & 2.07 & 100 & 6 & 21 & 4 & 0.405 & 1.332 & Lithium \\
\hline 5 & $\mathrm{f}$ & 75 & 7.23 & 100 & 8 & 39 & 7 & 0.571 & 24.822 & Valproic acid \\
\hline 6 & $\mathrm{f}$ & 45 & 4.22 & 100 & 6 & 21 & 12 & 0.214 & 7.346 & None \\
\hline 7 & $\mathrm{f}$ & 58 & 0.76 & 50 & 4 & 23 & 2 & 0.750 & 2.679 & Carbamazepine \\
\hline 8 & $\mathrm{f}$ & 75 & 2.93 & 100 & 6 & 31 & 3 & 0.667 & 0.037 & None \\
\hline 9 & $\mathrm{f}$ & 66 & 2.95 & 100 & 6 & 43 & 1 & 1.000 & 4.730 & None \\
\hline 10 & $\mathrm{f}$ & 46 & 2.89 & 100 & 6 & 36 & 2 & 0.810 & 0.858 & None \\
\hline 11 & $\mathrm{~m}$ & 50 & 1.62 & 100 & 6 & 37 & 10 & 0.643 & 0.661 & None \\
\hline 12 & $\mathrm{f}$ & 59 & 2.35 & 50 & 4 & 26 & 0 & 0.929 & 3.013 & Lithium \\
\hline
\end{tabular}


(A)

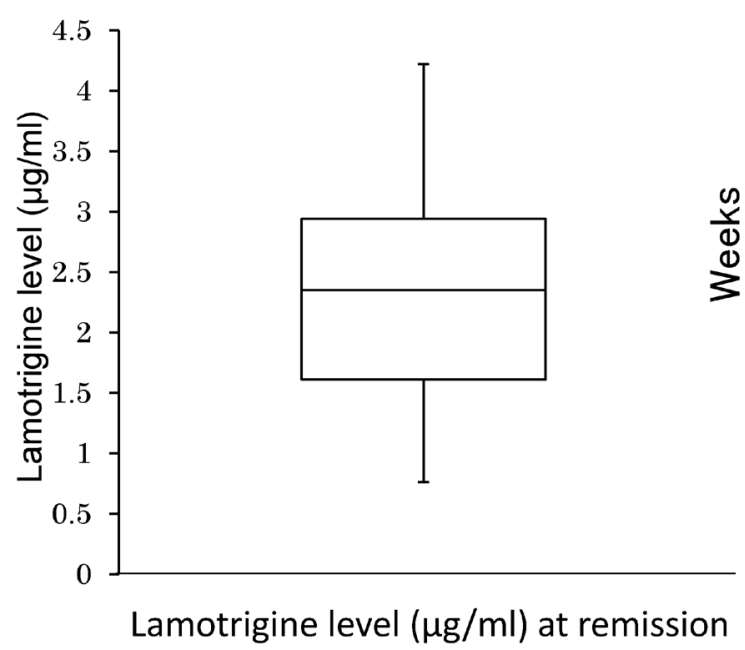

(B)

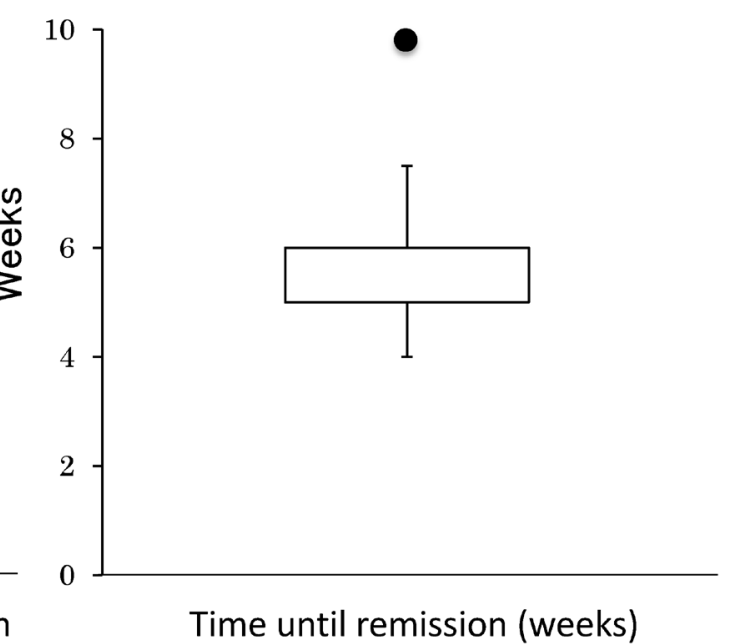

Fig. 2. Box Plots of the Patients' Serum Lamotrigine Levels ( $\mu \mathrm{g} / \mathrm{mL}$ ) at the Time of Remission (A) and the Time until Remission (Weeks) (B)

order) was reported to range from $5-11 \mu \mathrm{g} / \mathrm{mL},{ }^{23)}$ and another report suggested that a serum lamotrigine level of $12.7 \mu \mathrm{mol} / \mathrm{L}$ (about $3.2 \mu \mathrm{g} / \mathrm{mL}$ ) might represent a threshold for obtaining a good therapeutic response in treatment-resistant depressive disorders. ${ }^{24)}$ However, there have not been any reports about the therapeutic window of lamotrigine treatment for bipolar I disorder or differences in the efficacy of lamotrigine between bipolar I and II disorder. Furthermore, there is little information regarding appropriate serum levels of lamotrigine and the time until remission after the start of lamotrigine therapy in patients with bipolar II depression. In the present study, we examined the efficacy of lamotrigine and the serum lamotrigine level at the time of the remission of acute depression in bipolar II disorder patients.

Our findings indicate that when lamotrigine is administered to bipolar II disorder patients with acute depression a treatment response can be expected within 6 weeks. In addition, in the present study the median serum lamotrigine level at the time of the remission of acute depression (after excluding the patient that was treated with a combination of lamotrigine and valproic acid) was lower $(2.35 \mu \mathrm{g} / \mathrm{mL}$; range, $0.76-4.22 \mu \mathrm{g} / \mathrm{mL})$ than those described in previous reports. ${ }^{23,24)}$ This might be explained by the fact that this study was limited to patients with acute bipolar II depression. Furthermore, we evaluated the serum lamotrigine level after the remission of acute depression in bipolar II disorder patients. In general, the lamotrigine doses administered during the early stages of therapy are lower than those administered to prevent relapses during the remission maintenance phase. Moreover, a previous study reported that the mean lamotrigine dose was $256 \mathrm{mg}$ (range, $50-400 \mathrm{mg}$ ) during the remission maintenance phase, ${ }^{23)}$ whereas it was $91.7 \mathrm{mg}$ (range, $50-150 \mathrm{mg}$ ) in this study. Therefore, it is possible that the patients in our study did not receive maintenance doses of lamotrigine. Further studies examining the most effective levels of lamotrigine during the remission maintenance phase of bipolar II disorder are needed.

However, in the current study the mean time until the remission of acute depression was 6 weeks (range, 4-7.5 weeks), and it was reported that 8 weeks' lamotrigine monotherapy is an effective treatment for bipolar II depressive disorder. ${ }^{25}$ ) Thus, the results of the present study did not differ fundamentally from those obtained in previous studies of depressive bipolar II patients.

It is known that the co-administration of lamotrigine and valproic acid results in high lamotrigine levels. It has also been reported that the co-administration of lamotrigine and valproic acid reduced total lamotrigine clearance, whereas the renal clearance of lamotrigine remained unchanged. ${ }^{26,27)}$ In addition, the plasma elimination half-life and area under the curve of lamotrigine were increased. These alterations in the pharmacokinetics of lamotrigine were attributed to impaired glucuronide formation in the liver due to the competitive inhibition of hepatic enzymes by valproic acid. ${ }^{26,27)}$ In the present study, the patient that was co-administered valproic acid exhibited higher serum lamotrigine levels $(7.23 \mu \mathrm{g} / \mathrm{mL})$ than the other patients. Contrary to our expectations, the use of valproic acid did not affect the MADRS improvement rate. Importantly, it has been reported that the co-administration of valproic acid and lamotrigine might increase the risk of drug-induced skin eruptions, ${ }^{28}$ ) which can lead to the discontinuation of lamotrigine therapy. ${ }^{29)}$ In general, when skin eruptions occur after the co-administration of lamotrigine and valproic acid, we usually decide to discontinue the administration of lamotrigine because lamotrigine is the active drug rather than valproic acid. Furthermore, bipolar II depressive patients require long-term drug treatment. It is possible that in patients that are co-administered lamotrigine and valproic acid increased lamotrigine levels are associated with a high risk of skin eruptions. Therefore, the co-administration of valproic acid and lamotrigine for bipolar II is not recommended. In cases in which the co-administration of lamotrigine and valproic acid is considered necessary, the rate of increase in the dosage of lamotrigine should be slowed in order to prevent drug-induced skin eruptions.

It was reported that the side effects (cutaneous adverse reactions) of lamotrigine are affected by single nucleotide polymorphisms (e.g., human leukocyte antigen (HLA)-B*1502) and that HLA-B*1502 is associated with severe lamotrigineinduced cutaneous reactions. ${ }^{30)}$ However, HLA-B*1502 is not 
directly associated with lamotrigine-induced maculopapular exanthema in the Han Chinese population. ${ }^{31)}$ On the other hand, the factors involved in the regulation of the therapeutic effects of lamotrigine are poorly understood. Further studies are in progress to clarify these issues.

The limitations of this pilot study include its very small sample size, the lack of a placebo arm, and the lack of comparisons between responders and non-responders. Prospective studies involving larger sample sizes are required, and these studies should involve comparisons between the serum lamotrigine levels of responders and non-responders.

\section{CONCLUSION}

This study shows that the serum lamotrigine level is not a useful biomarker for predicting the therapeutic effect of lamotrigine or estimating appropriate lamotrigine doses for individual bipolar II depression patients. Furthermore, it is important to increase lamotrigine dosages carefully in order to prevent the occurrence of drug-induced skin eruptions. In addition, we consider that it is not appropriate to increase the dosage of lamotrigine simply to treat acute bipolar II depression because no correlation was detected between the improvement in the MADRS score and the serum lamotrigine level. Moreover, we consider that patients who do not exhibit any improvement even after being administered lamotrigine for 6 weeks and/or reaching a serum lamotrigine level of $>4.2 \mu \mathrm{g} / \mathrm{mL}$ are non-responders, and different treatment options should be considered for such patients.

Conflict of Interest The authors declare no conflict of interest.

\section{REFERENCES}

1) Judd LL, Akiskal HS. The prevalence and disability of bipolar spectrum disorders in the US population: re-analysis of the ECA database taking into account subthreshold cases. J. Affect. Disord., 73, 123-131 (2003)

2) Judd LL, Akiskal HS, Schettler PJ, Coryell W, Endicott J, Maser $\mathrm{JD}$, Solomon DA, Leon AC, Keller MB. A prospective investigation of the natural history of the long-term weekly symptomatic status of bipolar II disorder. Arch. Gen. Psychiatry, 60, 261-269 (2003).

3) Kessler RC, Berglund P, Demler O, Jin R, Merikangas KR, Walters EE. Lifetime prevalence and age-of-onset distributions of DSM-IV disorders in the National Comorbidity Survey Replication. Arch. Gen. Psychiatry, 62, 593-602 (2005).

4) Kessler RC, Akiskal HS, Angst J, Guyer M, Hirschfeld RM, Merikangas KR, Stang PE. Validity of the assessment of bipolar spectrum disorders in the WHO CIDI 3.0. J. Affect. Disord., 96, 259-269 (2006).

5) Calabrese JR, Bowden CL, Sachs GS, Ascher JA, Monaghan E, Rudd GD. A double-blind placebo-controlled study of lamotrigine monotherapy in outpatients with bipolar I depression. Lamictal 602 Study Group. J. Clin. Psychiatry, 60, 79-88 (1999).

6) Sharma V, Khan M, Corpse C. Role of lamotrigine in the management of treatment-resistant bipolar II depression: a chart review. $J$. Affect. Disord., 111, 100-105 (2008).

7) Ivković M, Damjanovic A, Jovanovic A, Cvetic T, Jasovic-Gasic M. Lamotrigine versus lithium augmentation of antidepressant therapy in treatment-resistant depression: efficacy and tolerability. Psychiatr. Danub., 21, 187-193 (2009).

8) van der Loos ML, Mulder PG, Hartong EG, Blom MB, Vergou- wen AC, de Keyzer HJ, Notten PJ, Luteijn ML, Timmermans MA, Vieta E, Nolen WA, LamLit Study Group. Efficacy and safety of lamotrigine as add-on treatment to lithium in bipolar depression: a multicenter, double-blind, placebo-controlled trial. J. Clin. Psychiatry, 70, 223-231 (2009).

9) Arif H, Svoronos A, Resor SR Jr, Buchsbaum R, Hirsch LJ. The effect of age and comedication on lamotrigine clearance, tolerability, and efficacy. Epilepsia, 52, 1905-1913 (2011).

10) Gilman JT. Lamotrigine: an antiepileptic agent for the treatment of partial seizures. Ann. Pharmacother., 29, 144-151 (1995).

11) Kusumakar V, Yatham LN. An open study of lamotrigine in refractory bipolar depression. Psychiatry Res., 72, 145-148 (1997).

12) Kilpatrick ES, Forrest G, Brodie MJ. Concentration-effect and concentration-toxicity relations with lamotrigine: a prospective study. Epilepsia, 37, 534-538 (1996).

13) Bartoli A, Guerrini R, Belmonte A, Alessandri MG, Gatti G, Perucca $\mathrm{E}$. The influence of dosage, age, and comedication on steady state plasma lamotrigine concentrations in epileptic children: a prospective study with preliminary assessment of correlations with clinical response. Ther. Drug Monit., 19, 252-260 (1997).

14) Chong E, Dupuis LL. Therapeutic drug monitoring of lamotrigine. Ann. Pharmacother., 36, 917-920 (2002).

15) Biton V. Pharmacokinetics, toxicology and safety of lamotrigine in epilepsy. Expert Opin. Drug Metab. Toxicol., 2, 1009-1018 (2006).

16) Kanner AM, Frey M. Adding valproate to lamotrigine: a study of their pharmacokinetic interaction. Neurology, 55, 588-591 (2000).

17) Mataringa MI, May TW, Rambeck B. Does lamotrigine influence valproate concentrations? Ther. Drug Monit., 24, 631-636 (2002).

18) Sbei M, Campellone JV. Stupor from lamotrigine toxicity. Epilepsia, 42, 1082-1083 (2001).

19) Goodwin GM, Bowden CL, Calabrese JR, Grunze H, Kasper S, White R, Greene P, Leadbetter R. A pooled analysis of 2 placebocontrolled 18-month trials of lamotrigine and lithium maintenance in bipolar I disorder. J. Clin. Psychiatry, 65, 432-441 (2004).

20) Bowden CL, Calabrese JR, Sachs G, Yatham LN, Asghar SA, Hompland M, Montgomery P, Earl N, Smoot TM, DeVeaugh-Geiss J, Lamictal 606 Study Group. A placebo-controlled 18-month trial of lamotrigine and lithium maintenance treatment in recently manic or hypomanic patients with bipolar I disorder. Arch. Gen. Psychiatry, 60, 392-400 (2003).

21) Calabrese JR, Suppes T, Bowden CL, Sachs GS, Swann AC, McElroy SL, Kusumakar V, Ascher JA, Earl NL, Greene PL, Monaghan ET. A double-blind, placebo-controlled, prophylaxis study of lamotrigine in rapid-cycling bipolar disorder. Lamictal 614 Study Group. J. Clin. Psychiatry, 61, 841-850 (2000).

22) Geddes JR, Calabrese JR, Goodwin GM. Lamotrigine for treatment of bipolar depression: independent meta-analysis and metaregression of individual patient data from five randomised trials. $\mathrm{Br}$. J. Psychiatry, 194, 4-9 (2009).

23) Katayama Y, Terao T, Kamei K, Hatano K, Kohno K, Makino M, Mizokami Y, Kodama K, Itoh H. Therapeutic window of lamotrigine for mood disorders: a naturalistic retrospective study. Pharmacopsychiatry, 47, 111-114 (2014).

24) Kagawa S, Mihara K, Nakamura A, Nemoto K, Suzuki T, Nagai G, Kondo T. Relationship between plasma concentrations of lamotrigine and its early therapeutic effect of lamotrigine augmentation therapy in treatment-resistant depressive disorder. Ther. Drug Monit., 36, 730-733 (2014)

25) Suppes T, Marangell LB, Bernstein IH, Kelly DI, Fischer EG, Zboyan HA, Snow DE, Martinez M, Al Jurdi R, Shivakumar G, Sureddi S, Gonzalez R. A single blind comparison of lithium and lamotrigine for the treatment of bipolar II depression. J. Affect. Disord., 111, 334-343 (2008).

26) Chapman A, Keane PE, Meldrum BS, Simiand J, Vernieres JC. Mechanism of anticonvulsant action of valproate. Prog. Neurobiol., 19, 315-359 (1982). 
27) Cohen AF, Land GS, Breimer DD, Yuen WC, Winton C, Peck AW. Lamotrigine, a new anticonvulsant: pharmacokinetics in normal humans. Clin. Pharmacol. Ther., 42, 535-541 (1987).

28) Ketter TA, Brooks JO, Hoblyn JC, Champion LM, Nam JY, Culver JL, Marsh WK, Bonner JC. Effectiveness of lamotrigine in bipolar disorder in a clinical setting. J. Psychiatr. Res., 43, 13-23 (2008).

29) Abe Y, Yasugawa S, Miyamoto K, Terao T. Valproate as a risk factor for lamotrigine discontinuation. J. Affect. Disord., 150, 1197-1199 (2013).
30) Man CB, Kwan P, Baum L, Yu E, Lau KM, Cheng AS, Ng MH. Association between HLA-B*1502 allele and antiepileptic drug-induced cutaneous reactions in Han Chinese. Epilepsia, 48, 1015-1018 (2007).

31) An DM, Wu XT, Hu FY, Yan B, Stefan H, Zhou D. Association study of lamotrigine-induced cutaneous adverse reactions and HLA-B*1502 in a Han Chinese population. Epilepsy Res., 92, $226-230(2010)$ 